\title{
Editorial on 'Estimation of Myocardial Viability in Patients with Ischaemic Mitral Regurgitation' (Morgan et al.)
}

\author{
Shubhra Sinha, Hunaid A. Vohra \\ Bristol Heart Institute, University Hospitals Bristol, Bristol, UK \\ Correspondence to: Hunaid A. Vohra. Bristol Heart Institute, University Hospitals, Bristol, UK. Email: hunaid.vohra@UHBristol.nhs.uk. \\ Comment on: Morgan AE, Zhang Y, Tartibi M, et al. Ischemic Mitral Regurgitation: Abnormal Strain Overestimates Nonviable Myocardium. Ann \\ Thorac Surg 2018;105:1754-61.
}

Submitted Sep 14, 2018. Accepted for publication Sep 19, 2018.

doi: $10.21037 /$ jtd.2018.09.104

View this article at: http://dx.doi.org/10.21037/jtd.2018.09.104

Ischaemic mitral regurgitation (IMR) is a common finding in the context of coronary artery disease with some studies showing it to be present in $10 \%$ of these patients $(1,2)$. It is associated with decreased long-term survival post-MI and post-revascularisation (3). The mainstay of treatment at present consists of myocardial revascularisation. However, the need for concomitant intervention on the mitral valve in those patients with moderate IMR remains an area of much debate in the cardiac surgical community. One of the many factors to consider in this context is the viability of the left ventricular (LV) myocardium and its inherent ability for reverse remodelling. In this study by Morgan et al. (4), a direct comparison is made between using $\mathrm{LV}$ strain and late gadolinium enhancement (LGE), as markers of the same. They propose that when using cardiac magnetic resonance imaging (cMRI) to assess the myocardium in patients with greater than mild MR, abnormal strain overestimates the number of non-viable LV segments as compared to LGE.

As our readers are aware, IMR occurs following a myocardial infarction (MI) due to decreased LV contraction and displacement of the papillary muscle resulting in tethering of the leaflets. This results in a displacement of the leaflet coaptation point towards the apex and a Carpentier type IIIb defect. Thus, it is not an inherent problem of the valvular apparatus but of the ventricle. Therefore, the primary mechanism of intervention is to aid the reverse remodelling of the $\mathrm{LV}$ via coronary revascularisation. But the question remains about whether we should intervene on the mitral valve at the same operation. Three randomised trials have attempted to answer this question (5-7). The two earlier trials $(5,6)$ did show improvement in markers of ventricular reverse remodelling and New York Heart Association (NYHA) class in those patients that underwent CABG with mitral valve repair ( $M V r)$ but no difference in survival in the short-term. However, a large multicentre trial conducted by the Cardiothoracic Surgical Trials Network (7) comparing CABG alone with CABG and MV surgery showed no difference in the survival, $L V$ end-systolic volume index, NYHA class nor incidence of major adverse cardiac and cerebrovascular events. In fact, they showed a greater number of neurological events and supraventricular tachycardias in the group having concomitant MV surgery. As you would expect there was less residual MR post-operatively in those having CABG with MV surgery but even in the CABG alone group 70\% had no residual MR. They also demonstrated a significantly higher rate of recurrence of moderate or severe $M R$ in the group undergoing repair as opposed to replacement. Our meta-analysis (8) looked at 11 papers consisting of a total of 1,406 patients with moderate IMR. They found a greater improvement in grade of MR and LV systolic diameter in patients undergoing $\mathrm{MVr}$ in addition to $\mathrm{CABG}$ as opposed to $\mathrm{CABG}$ alone. However, there was no difference in postoperative NYHA class, ejection fraction (EF) or survival. This goes to show that we still need greater clarity on which of our patients will benefit from mitral intervention at the time of CABG.

As is often the case, the answer is 'It depends'. Several factors need to be considered. Patient factors can determine tolerance of a longer operation, such as their age, presence of co-morbidities and LVEF. Another key factor is the likelihood of the ventricle to recover from the ischaemic 
insult. If the myocardium is stunned but has the ability to recover on optimising its vascular supply there may be an argument to proceed with CABG alone or perform MV repair rather than $\mathrm{MV}$ replacement. However, if there is a large area of myocardial necrosis, difficulty in achieving full revascularisation or there is gross dilatation of the $L V$ then concomitant MV replacement may be considered.

Several imaging modalities have been utilised to assess myocardial viability, including cMRI. Both LV strain $(9,10)$ and LGE measurements $(11,12)$ have been shown to correlate with the degree of viability. The use of viability studies in assessing coronary artery disease patients with $\mathrm{LV}$ dysfunction has been studied in recent trials $(13,14)$ and an earlier meta-analysis (15), with mixed results. The meta-analysis of over 3,000 patients by Allman et al. (15) showed a strong correlation between identification of viability on non-invasive testing and improved survival following revascularisation. The PARR-2 study (13) looked at the use of positron emission tomography (PET) in guiding the management of patients with severe $\mathrm{LV}$ dysfunction and suspected coronary artery disease. It did not show any improvement in clinical outcomes with PET-guided therapy however in nearly a quarter of cases the PET-based advice was not adhered to. Subsequent analysis has shown that in the subset in whom the advice was adhered to, there was an improvement in the clinical outcome. The viability sub-study of the STICH trial (14) found a correlation between myocardial viability and revascularisation which was statistically significant in the univariate analysis but not on multivariable analysis. Therefore, at present the European Society of Cardiology guidelines state that ischaemia and viability testing in patients with coronary disease and heart failure is a Class IIb Level of Evidence B recommendation (16). There are presently no specific guidelines for the use of these studies in the context of IMR.

In the study by Morgan et al. (4), analysis was done on cMRI data on 16 patients with greater than mild MR and 7 normal volunteers. They measured longitudinal and circumferential strain, LGE and stress perfusion. They were able to show that in their cohort of patients $7.4 \%$ of LV segments had transmural infarcts (TMI), defined as hyperenhancement of $>50 \%$ of wall thickness, as per LGE. Whereas $>14.5 \%$ of LV segments were non-viable by strain thresholds (between 1-2.5 standard deviations from normal mean). They found impaired strain in the segments immediately bordering the TMI. These segments though ischaemic may have hibernating myocardium with potential for reverse remodelling and return to normal contractility. Strain was also globally impaired in IMR patients as compared with normal subjects. Therefore, LV strain is likely to over-estimate the number of non-viable segments when compared with LGE in patients with IMR. Though the sample size is small, this study shows the potential importance of using LGE in the assessment of patients with IMR. Uniquely, it also provides a direct comparison between 2 commonly used parameters of viability and would form the foundation of a bigger multi-centre randomised controlled trial.

As cardiac surgeons and cardiologists we will continue to debate the timing and type of surgical intervention indicated in patients with moderate IMR. Viability assessment may play a role in answering this question. This paper has provided some insight into the use of cMRI, and specifically LGE, in determining myocardial viability in this patient cohort. However, larger studies with suitable followup and clinical correlation are needed to clarify the role of this assessment modality. This may apply not only to IMR but also to prediction of post-operative contractility after surgery for other pathological entities like degenerative MR and severe aortic stenosis.

\section{Acknowledgements}

None.

\section{Footnote}

Conflicts of Interest: The authors have no conflicts of interest to declare.

\section{References}

1. Bursi F, Enriquez-Sarano M, Nkomo VT, et al. Heart failure and death after myocardial infarction in the community: the emerging role of mitral regurgitation. Circulation 2005;111:295-301.

2. Pérez de Isla L, Zamorano J, Quezada M, et al. Functional mitral regurgitation after a first non-ST-segment elevation acute coronary syndrome: contribution to congestive heart failure. Eur Heart J 2007;28:2866-72.

3. Lamas GA, Mitchell GF, Flaker GC, et al. Clinical significance of mitral regurgitation after acute myocardial infarction. Survival and Ventricular Enlargement Investigators. Circulation 1997;96:827-33.

4. Morgan AE, Zhang Y, Tartibi M, et al. Ischemic Mitral 
Regurgitation: Abnormal Strain Overestimates Nonviable Myocardium. Ann Thorac Surg 2018;105:1754-61.

5. Chan KM, Punjabi PP, Flather M, et al. Coronary artery bypass surgery with or without mitral valve annuloplasty in moderate functional ischemic mitral regurgitation: final results of the Randomized Ischemic Mitral Evaluation (RIME) trial. Circulation 2012;126:2502-10.

6. Fattouch K, Guccione F, Sampognaro R, et al. POINT: Efficacy of adding mitral valve restrictive annuloplasty to coronary artery bypass grafting in patients with moderate ischemic mitral valve regurgitation: a randomized trial. J Thorac Cardiovasc Surg 2009;138:278-85.

7. Michler RE, Smith PK, Parides MK, et al. Two-Year Outcomes of Surgical Treatment of Moderate Ischemic Mitral Regurgitation. N Engl J Med 2016;374:1932-41.

8. Salmasi MY, Harky A, Chowdhury MF, et al. Should the mitral valve be repaired for moderate ischemic mitral regurgitation at the time of revascularization surgery? J Card Surg 2018;33:374-84.

9. Lancaster TS, Kar J, Cupps BP, et al. Topographic mapping of left ventricular regional contractile injury in ischemic mitral regurgitation. J Thorac Cardiovasc Surg 2017;154:149-58.e1.

10. Cupps BP, Bree DR, Wollmuth JR, et al. Myocardial viability mapping by magnetic resonance-based multiparametric systolic strain analysis. Ann Thorac Surg

Cite this article as: Sinha S, Vohra HA. Editorial on 'Estimation of Myocardial Viability in Patients with Ischaemic Mitral Regurgitation' (Morgan et al.). J Thorac Dis 2018;10(Suppl 33):S4073-S4075. doi: 10.21037/jtd.2018.09.104
2008;86:1546-53.

11. Kim RJ, Wu E, Rafael A, et al. The use of contrastenhanced magnetic resonance imaging to identify reversible myocardial dysfunction. $\mathrm{N}$ Engl J Med 2000;343:1445-53.

12. Kim RJ, Fieno DS, Parrish TB, et al. Relationship of MRI delayed contrast enhancement to irreversible injury, infarct age, and contractile function. Circulation 1999;100:1992-2002.

13. Beanlands RS, Nichol G, Huszti E, et al. F-18fluorodeoxyglucose positron emission tomography imaging-assisted management of patients with severe left ventricular dysfunction and suspected coronary disease: a randomized, controlled trial (PARR-2). J Am Coll Cardiol 2007;50:2002-12.

14. Bonow RO, Maurer G, Lee KL, et al. Myocardial viability and survival in ischemic left ventricular dysfunction. $\mathrm{N}$ Engl J Med 2011;364:1617-25.

15. Allman KC, Shaw LJ, Hachamovitch R, et al. Myocardial viability testing and impact of revascularization on prognosis in patients with coronary artery disease and left ventricular dysfunction: a meta-analysis. J Am Coll Cardiol 2002;39:1151-8.

16. Sousa-Uva M, Neumann FJ, Ahlsson A, et al. 2018 ESC/ EACTS Guidelines on myocardial revascularization. Eur J Cardiothorac Surg 2018. [Epub ahead of print]. 